\title{
Maximising cost-effectiveness and minimising waste in modern ocular surgical simulation
}

\author{
Jennifer Hind $\mathbb{B}^{1}$ - Magdalena Edington ${ }^{1}$ - David Lockington ${ }^{1}{ }^{1}$
}

Received: 27 July 2020 / Revised: 14 August 2020 / Accepted: 17 August 2020 / Published online: 25 August 2020

(c) The Royal College of Ophthalmologists 2020

\section{To the Editor:}

In the UK, the ophthalmic specialist curriculum mandates the use of surgical simulation in training [1]. As ophthalmologists return to surgical sessions following COVID-19 restrictions, the benefits of learning and maintaining skills through simulation have been increasingly recognised by juniors and seniors alike [2]. Ophthalmic surgical simulation has previously been demonstrated to be cost-effective and cost-saving due to reduced surgical complication rates in cataract surgery by junior trainees [3]. The benefits of promoting greater simulation use in training are obvious, but this new culture has not previously been reflected in traditional resource allocation in ophthalmology training programmes. An increasing range of model eyes for simulation are available, of varying complexity and expense $[4,5]$. It should be recognised that any effective simulation programme will not only have initial set up costs (microscopes, equipment), but will require an ongoing training investment, through the recurrent purchase of necessary consumables (model eyes, suture materials).

There are currently $28 \mathrm{ST}$ training posts in the West of Scotland. Following the recent creation of a bespoke ocular simulation suite, we purchased 80 model eyes ( 8 boxes $\times$ 10 eyes) with an initial outlay of $£ 1518$ (Phillips Studio, Bristol, UK). We realised that our resources would be quickly depleted if the model eyes were only used for their advertised purpose. To maximise use and minimise costs of these simulated model eyes, we allocated three eyes per trainee and promoted a "single eye, multiple procedures" concept. To prevent a "use once and throw out" mentality, we challenged the trainees to see "how many procedures

Jennifer Hind

jennlhind@gmail.com

1 Tennent Institute of Ophthalmology, Gartnavel General Hospital, 1053 Great Western Road, Glasgow G12 0YN, UK can you perform on a model eye?". The winning entry (Figs. 1 and 2) demonstrates 49 procedures performed by a single trainee on a single corneal suturing eye. This included limbal traction sutures; cataract wounds; scleral flaps; tectonic grafts and penetrating injury repairs, demonstrating imaginative use of simulation equipment to achieve multiple outcomes, and saving an estimated $£ 200$ in the process. We would encourage other training programmes to exploit the healthy competitive streak found amongst many trainees to optimise surgical training in this cost-effective way, while maintaining the sustainability of simulation training.

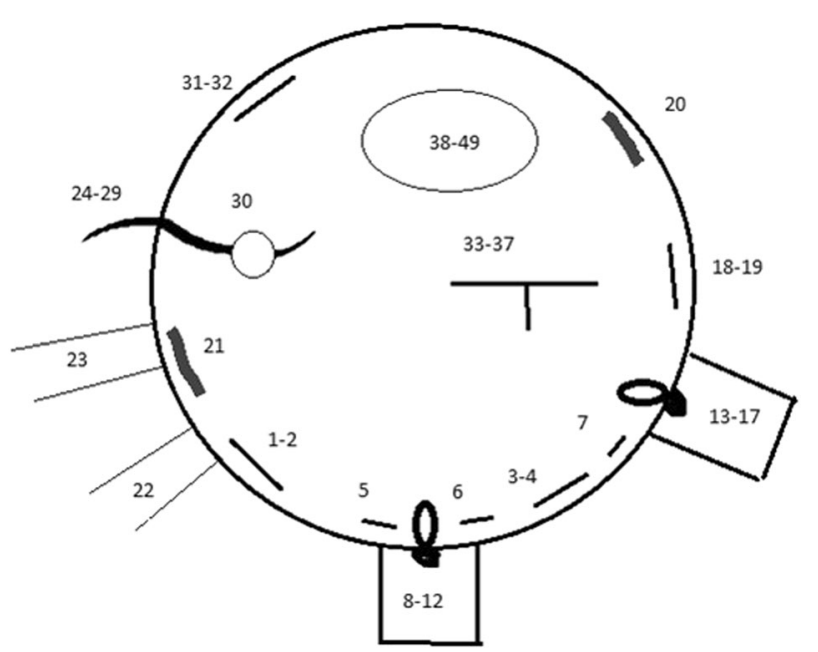

Fig. 1 Diagram of single model eye with simulated surgical procedures performed as described. 1-2: phacoemulsification (phaco) wound and suture, 3-4: phaco wound and suture, 5-6: partial thickness corneal relaxing incisions, 7: paracentesis, 8-12: scleral flap, scleral punch, surgical iridotomy, $2 \times$ adjustable suture, 13-17: scleral flap, scleral punch, surgical iridotomy, $2 \times$ permanent suture, 18-19: phaco wound and suture, 20-21: limbal traction sutures, 22-23: scleral tunnels, 24-29: penetrating injury with foreign body repair (iris FB removal, iris suture 7-0 01 , corneal suture $1-0 \times 3$, limbal suture), 30 : corneal glue, 31-32: phaco wound and suture, 33-37: corneal laceration repair ( $5 \times$ suture), $38-49$ : tectonic graft ( $12 \times$ suture). 
Fig. 2 Photograph of the winning entry. a Macroscopic photo of corneal suturing eye with the above procedures. b Microscopic photograph of corneal suturing eye showing tectonic graft superiorly; corneal laceration repair centrally and scleral flap with iridotomy inferiorly.

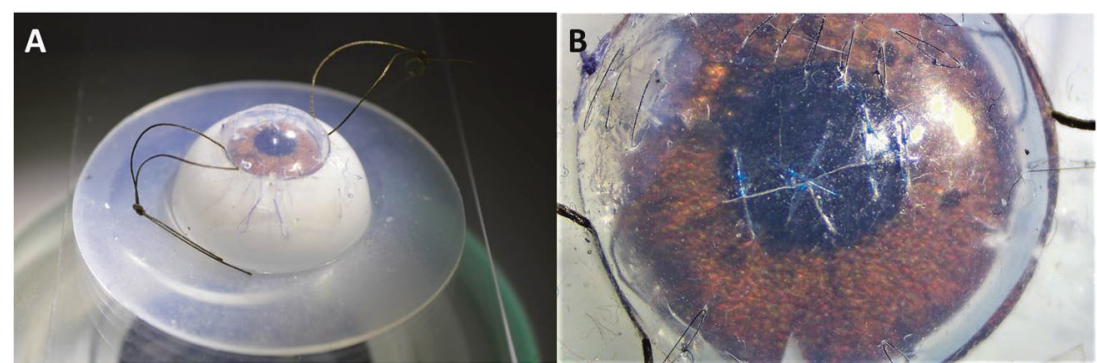

\section{Compliance with ethical standards}

Conflict of interest The authors declare that they have no conflict of interest.

Publisher's note Springer Nature remains neutral with regard to jurisdictional claims in published maps and institutional affiliations.

\section{References}

1. Royal College of Ophthalmologists. Simulation in Training. London. 2018. Accessed 10 Jun 2020. www.rcophth.ac.uk/training/ ostinformation/simulation.
2. Thomsen ASS, Bach-Holm D, Kjærbo H, Højgaard-Olsen K, Subhi Y, Saleh GM, et al. Operating room performance improves after proficiency-based virtual reality cataract surgery training. Ophthalmology. 2017;124:524-31.

3. Ferris JD, Donachie PH, Johnston RL, Barnes B, Olaitan M, Sparrow JM. Royal College of Ophthalmologists' National Ophthalmology Database study of cataract surgery: report 6 . The impact of EyeSi virtual reality training on complications rates of cataract surgery performed by first and second year trainees. $\mathrm{Br} \mathbf{J}$ Ophthalmol. 2020;104:324-9.

4. Hom-Choudhury, A, Innes, JR. The Hull Anterior Vitrectomy Simulation System (HAVSS): a validated novel simulation for training in the management of intra-operative vitreous loss. Eye. 2020. https://doi.org/10.1038/s41433-020-0772-9.

5. Phillips Studio Eye. Ophthalmic Simulated Surgery. Bristol. Accessed 23 Jul 2020. www.phillipsstudio.co.uk. 